\title{
Some majorization integral inequalities for functions defined on rectangles
}

\author{
Shanhe Wu', Muhammad Adil Khan², Abdul Basir² and Reza Saadati ${ }^{*}$
}

\author{
Correspondence: rsaadati@eml.cc \\ ${ }^{3}$ Department of Mathematics, Iran \\ University of Science and \\ Technology, Tehran, Iran \\ Full list of author information is \\ available at the end of the article
}

\begin{abstract}
In this paper, we first prove an integral majorization theorem related to integral inequalities for functions defined on rectangles. We then apply the result to establish some new integral inequalities for functions defined on rectangles. The results obtained are generalizations of weighted Favard's inequality, which also provide a generalization of the results given by Maligranda et al. (J. Math. Anal. Appl. 190:248-262, 1995) in an earlier paper.
\end{abstract}

MSC: 26A51;26D15; 26D20

Keywords: Convex function; Coordinate convex function; Rectangle; Majorization; Favard's inequality

\section{Introduction}

There is a certain intuitive appeal to the vague notion that the components of an $n$-tuple $\mathbf{x}$ are less spread out, or more nearly equal, than the components of an $n$-tuple $\mathbf{y}$. The notion arises in a variety of contexts, and it can be made precise in a number of ways. In remarkably many cases, the appropriate statement is that $\mathbf{x}$ is majorized by $\mathbf{y}$ (or $\mathbf{y}$ majorizes $\mathbf{x}$ ). Namely, for two $n$-tuples $\mathbf{x}=\left(x_{1}, x_{2}, \ldots, x_{n}\right)$ and $\mathbf{y}=\left(y_{1}, y_{2}, \ldots, y_{n}\right), \mathbf{x}$ is said to be majorized by $\mathbf{y}$ (denoted $\mathbf{x} \prec \mathbf{y}$ ) if $\sum_{i=1}^{m} x_{[i]} \leq \sum_{i=1}^{m} y_{[i]}$ for $m=1,2, \ldots, n-1$ and $\sum_{i=1}^{n} x_{i}=\sum_{i=1}^{n} y_{i}$, where $x_{[1]} \geq x_{[2]} \geq \cdots \geq x_{[n]}$ and $y_{[1]} \geq y_{[2]} \geq \cdots \geq y_{[n]}$ are rearrangements of $\mathbf{x}$ and $\mathbf{y}$ in descending order. A mathematical origin of majorization is illustrated by the work of Schur [35] on Hadamard's determinant inequality. Many mathematical characterization problems are known to have solutions that involve majorization. Complete and superb references on the subject are the books [9, 28]. The comprehensive survey by Ando [7] provides alternative derivations, generalizations, and a different viewpoint.

The following theorem is known in the literature as the majorization theorem (see [20, $22,23,33,35])$.

Theorem 1.1 Let $f: I \rightarrow \mathbb{R}$ be a continuous convex function on the interval $I$, and let $\mathbf{x}=\left(x_{1}, x_{2}, \ldots, x_{n}\right)$ and $\mathbf{y}=\left(y_{1}, y_{2}, \ldots, y_{n}\right)$ be two $n$-tuples such that $x_{i}, y_{i} \in I(i=1,2, \ldots, n)$. If $\mathbf{x}$ is majorized by $\mathbf{y}$, then

$$
\sum_{i=1}^{n} f\left(x_{i}\right) \leq \sum_{i=1}^{n} f\left(y_{i}\right)
$$

\section{Springer}


The inequality asserted by Theorem 1.1 is also called majorization inequality. It is an inequality in elementary algebra for convex real-valued functions defined on an interval of the real line, and it generalizes the finite form of Jensen's inequality. This majorization ordering is equivalently described in Kemperman's review [25]. An extension of this fact for arbitrary real weights and decreasing $n$-tuples $\mathbf{x}$ and $\mathbf{y}$ can be found in [19]. General results of this type are obtained by Dragomir [17] and Niezgoda [30]. In recent years, many formulas such as Taylor formula, Hermite interpolating, Montgommery identities and inequalities for means, etc. have been used and generalized by majorization inequalities for $n$-convex functions; see [1-8, 10-15, 21, 24, 29, 31, 36, 37, 41-45] and references therein.

Recently, it has come to our attention that certain integral majorization theorems, we begin with recalling some relevant results. In 1947, Fuchs [19] gave the following integral majorization theorem for convex functions and two monotonic sequences.

Theorem $1.2([19])$ Let $\kappa(\tau), v(\tau):[a, b] \rightarrow \mathbb{R}$ be continuous and increasing functions, and let $\mu:[a, b] \rightarrow \mathbb{R}$ be a function of bounded variation.

(i) If

$$
\int_{x}^{b} \kappa(\tau) d \mu(\tau) \leq \int_{x}^{b} \nu(\tau) d \mu(\tau) \quad \text { for } x \in[a, b]
$$

and

$$
\int_{a}^{b} \kappa(\tau) d \mu(\tau)=\int_{a}^{b} \nu(\tau) d \mu(\tau)
$$

then for every continuous convex function $\phi$, we have

$$
\int_{a}^{b} \phi[\kappa(\tau)] d \mu(\tau) \leq \int_{a}^{b} \phi[v(\tau)] d \mu(\tau) .
$$

(ii) If

$$
\int_{x}^{b} \kappa(\tau) d \mu(\tau) \leq \int_{x}^{b} v(\tau) d \mu(\tau) \quad \text { for } x \in[a, b]
$$

then for every continuous increasing convex function $\phi$, we have

$$
\int_{a}^{b} \phi[\kappa(\tau)] d \mu(\tau) \leq \int_{a}^{b} \phi[v(\tau)] d \mu(\tau) .
$$

In 1995, Maligranda, Pečarić, and Persson [27] established the following analogue of the Fuchs inequality.

Theorem 1.3 ([27]) Let $w$ be a weight function, and let $f$ and $g$ be positive integrable functions on $[a, b]$. Suppose that $\varphi:[0, \infty) \rightarrow \mathbb{R}$ is a convex function and that

$$
\int_{a}^{x} f(t) w(t) d t \leq \int_{a}^{x} g(t) w(t) d t \quad \text { for } x \in[a, b]
$$


and

$$
\int_{a}^{b} f(t) w(t) d t=\int_{a}^{b} g(t) w(t) d t
$$

(i) Iff is a decreasing function on $[a, b]$, then

$$
\int_{a}^{b} \varphi[f(t)] w(t) d t \leq \int_{a}^{b} \varphi[g(t)] w(t) d t
$$

(ii) If $g$ is an increasing function on $[a, b]$, then

$$
\int_{a}^{b} \varphi[g(t)] w(t) d t \leq \int_{a}^{b} \varphi[f(t)] w(t) d t
$$

In 1933, Favard [18] proved the following results.

Theorem 1.4 Let $\Phi$ be a nonnegative continuous concave function on $[a, b]$, not identically zero, and let $\Upsilon$ be a convex function on $[0, \widetilde{\Phi}]$, where

$$
\widetilde{\Phi}=\frac{2}{b-a} \int_{a}^{b} \Phi(x) d x
$$

Then

$$
\frac{1}{b-a} \int_{a}^{b} \Upsilon[\Phi(x)] d x \leq \int_{0}^{1} \Upsilon(s \widetilde{\Phi}) d s
$$

As a consequence of Theorem 1.4, the following inequality was also established in [18].

Theorem 1.5 Let $\Phi$ be a concave nonnegative function on $[a, b] \subset \mathbb{R}$. If $q>1$, then

$$
\frac{1}{b-a} \int_{a}^{b} \Phi^{q}(x) d x \leq \frac{2^{q}}{q+1}\left(\frac{1}{b-a} \int_{a}^{b} \Phi(x) d x\right)^{q} .
$$

Maligranda, Pečarić, and Persson [27] gave the following generalization of the Favard inequality.

\section{Theorem 1.6 ([27])}

(i) Let $\Phi$ be a positive increasing concave function on $[a, b]$, and let $\Upsilon$ be a convex function on $[0, \widetilde{\Phi}]$, where

$$
\widetilde{\Phi}=(b-a) \int_{a}^{b} \Phi(t) w(t) d t / \int_{a}^{b}(t-a) w(t) d t
$$

Then

$$
\frac{1}{b-a} \int_{a}^{b} \Upsilon[\Phi(t)] w(t) d t \leq \int_{0}^{1} \Upsilon(s \widetilde{\Phi}) w[a(1-s)+b s] d s
$$


(ii) Let $\Phi$ be a positive decreasing concave function on $[a, b]$, and let $\Upsilon$ be a convex function on $[0, \widetilde{\Phi}]$, where

$$
\widetilde{\Phi}=(b-a) \int_{a}^{b} \Phi(t) w(t) d t / \int_{a}^{b}(b-t) w(t) d t
$$

Then

$$
\frac{1}{b-a} \int_{a}^{b} \Upsilon[\Phi(t)] w(t) d t \leq \int_{0}^{1} \Upsilon(s \widetilde{\Phi}) w[a s+b(1-s)] d s
$$

For further results related to generalizations, extensions, and refinements of the integral inequalities of majorization type, we refer the reader to [1-6, 26, 28, 32, 34, 38-40, 46-48]. In this paper, we extend majorization and Favard inequalities from functions defined on intervals to functions defined on rectangles. The results presented in this paper generalize the results of Maligranda, Pečarić, and Persson [27].

\section{Preliminaries}

In this section, we introduce some notions and lemmas.

Definition 2.1 A function $\phi: \Omega \rightarrow \mathbb{R}$ defined on a convex subset $\Omega$ of $\mathbb{R}^{n}$ is said to be convex if

$$
\phi(\lambda \mathbf{x}+(1-\lambda) \mathbf{y}) \leq \lambda \phi(\mathbf{x})+(1-\lambda) \phi(\mathbf{y})
$$

for all $\mathbf{x}, \mathbf{y} \in \Omega$ and $\lambda \in[0,1]$.

In this paper, convex functions considered are supposed to be twice differentiable. It is well know that if the function $\phi$ is convex, then

$$
\phi(\mathbf{x})-\phi(\mathbf{y}) \geq \nabla_{+} \phi(\mathbf{y})(\mathbf{x}-\mathbf{y}) \quad \text { for all } \mathbf{x}, \mathbf{y} \in \Omega,
$$

where

$$
\nabla_{+} \phi(\mathbf{y})(\mathbf{x}-\mathbf{y})=\left\langle\frac{\partial \phi_{+}(\mathbf{y})}{\partial \mathbf{y}},(\mathbf{x}-\mathbf{y})\right\rangle, \quad \frac{\partial \phi_{+}(\mathbf{y})}{\partial \mathbf{y}}=\left(\frac{\partial \phi_{+}(\mathbf{y})}{\partial y_{1}}, \frac{\partial \phi_{+}(\mathbf{y})}{\partial y_{2}}, \ldots, \frac{\partial \phi_{+}(\mathbf{y})}{\partial y_{n}}\right)
$$

$\mathbf{x}=\left(x_{1}, x_{2}, \ldots, x_{n}\right), \mathbf{y}=\left(y_{1}, y_{2}, \ldots, y_{n}\right) \in \Omega$, and $\langle\cdot, \cdot \cdot\rangle$ is the usual inner product in $\mathbb{R}^{n}$.

In the literature, there are many generalizations of convex functions in different directions. One of them is coordinate convex functions introduced by Dragomir [16].

Definition 2.2 ([16]) Let us consider a bidimensional interval $S=[a, b] \times[c, d] \subset \mathbb{R}^{2}$. A function $\phi: S \rightarrow \mathbb{R}$ is said to be coordinate convex if the partial functions $\phi_{y}:[a, b] \rightarrow \mathbb{R}$ defined as $\phi_{y}(u)=\phi(u, y)$ and $\phi_{x}:[c, d] \rightarrow \mathbb{R}$ defined as $\phi_{x}(v)=\phi(x, v)$ are convex where defined for all $y \in[c, d]$ and $x \in[a, b]$.

Lemma 2.3 ([16]) Every convex function $\phi: S \rightarrow \mathbb{R}$ is coordinate convex.

Lemma 2.4 ([27]) Let $v$ be a weight function on $[a, b]$. 
(i) If $h$ is a decreasing function on $[a, b]$, then

$$
\int_{a}^{b} h(t) v(t) d t \int_{a}^{x} v(t) d t \leq \int_{a}^{x} h(t) v(t) d t \int_{a}^{b} v(t) d t \quad \text { for } x \in[a, b] .
$$

(ii) If $h$ is an increasing function on $[a, b]$, then

$$
\int_{a}^{x} h(t) v(t) d t \int_{a}^{b} v(t) d t \leq \int_{a}^{b} h(t) v(t) d t \int_{a}^{x} v(t) d t \quad \text { for } x \in[a, b] .
$$

\section{Majorization inequalities for functions defined on rectangles}

In this section, we establish some majorization integral inequalities for functions defined on rectangles. The following theorem is a generalization of Theorem 1.3 mentioned in the Introduction.

Theorem 3.1 Let $w$ and $p$ be positive continuous functions on $[a, b]$ and $[c, d]$ respectively, and let $f, g$ and $h, k$ be positive differentiable functions on $[a, b]$ and $[c, d]$ respectively. Suppose that $\phi:[0, \infty) \times[0, \infty) \rightarrow \mathbb{R}$ is a convex function and that

$$
\begin{aligned}
& \int_{a}^{x} g(t) w(t) d t \leq \int_{a}^{x} f(t) w(t) d t \quad \text { for } x \in[a, b] \\
& \int_{c}^{y} k(s) p(s) d s \leq \int_{c}^{y} h(s) p(s) d s \quad \text { for } y \in[c, d] \\
& \int_{a}^{b} g(t) w(t) d t=\int_{a}^{b} f(t) w(t) d t
\end{aligned}
$$

and

$$
\int_{c}^{d} k(s) p(s) d s=\int_{c}^{d} h(s) p(s) d s
$$

(i) If $g$ and $k$ are decreasing functions on $[a, b]$ and $[c, d]$ respectively, then

$$
\int_{a}^{b} \int_{c}^{d} \phi(g(t), k(s)) w(t) p(s) d t d s \leq \int_{a}^{b} \int_{c}^{d} \phi(f(t), h(s)) w(t) p(s) d t d s
$$

(ii) Iff and $h$ are increasing functions on $[a, b]$ and $[c, d]$ respectively, then

$$
\int_{a}^{b} \int_{c}^{d} \phi(f(t), h(s)) w(t) p(s) d t d s \leq \int_{a}^{b} \int_{c}^{d} \phi(g(t), k(s)) w(t) p(s) d t d s
$$

Proof

(i) Since $\phi:[0, \infty) \times[0, \infty) \rightarrow \mathbb{R}$ is a convex function, we have

$$
\phi(x, y)-\phi(w, z) \geq\left\langle\nabla_{+} \phi(w, z),(x-w, y-z)\right\rangle, \quad \forall(x, y),(w, z) \in[0, \infty) \times[0, \infty)
$$




$$
\Longleftrightarrow \phi(x, y)-\phi(w, z) \geq \frac{\partial \phi_{+}(w, z)}{\partial w}(x-w)+\frac{\partial \phi_{+}(w, z)}{\partial z}(y-z) .
$$

Put $x=f(t), y=h(s), w=g(t), z=k(s)$ in the last inequality and assume that

$$
\begin{array}{ll}
\psi_{s}^{1}(t)=\left.\frac{\partial \phi(u, v)}{\partial u}\right|_{u=g(t), v=k(s)}, & \psi_{s}^{3}(t)=\left.\frac{\partial^{2} \phi(u, v)}{\partial^{2} u}\right|_{u=g(t), v=k(s)}, \\
\psi_{s}^{2}(t)=\left.\frac{\partial \phi(u, v)}{\partial v}\right|_{u=g(t), v=k(s)}, & \psi_{s}^{4}(t)=\left.\frac{\partial^{2} \phi(u, v)}{\partial^{2} v}\right|_{u=g(t), v=k(s)} .
\end{array}
$$

Then we have

$$
\phi(f(t), h(s))-\phi(g(t), k(s)) \geq \psi_{s}^{1}(t)(f(t)-g(t))+\psi_{s}^{2}(t)(h(s)-k(s)) .
$$

Set

$$
F(x)=\int_{a}^{x}(f(t)-g(t)) w(t) d t, \quad x \in[a, b]
$$

and

$$
G(y)=\int_{c}^{y}(h(s)-k(s)) p(s) d s, \quad y \in[c, d] .
$$

Then, from the assumptions in Theorem 3.1 we conclude that

$$
\begin{aligned}
& F(x) \geq 0, \quad G(y) \geq 0 \quad \text { for } x \in[a, b], y \in[c, d], \\
& F(a)=F(b)=G(c)=G(d)=0 .
\end{aligned}
$$

Multiplying both sides of inequality (3.3) by $w(t) p(s)$, we get

$$
\begin{aligned}
& {[\phi(f(t), h(s))-\phi(g(t), k(s))] w(t) p(s)} \\
& \quad \geq \psi_{s}^{1}(t)[f(t)-g(t)] w(t) p(s)+\psi_{s}^{2}(t)[h(s)-k(s)] w(t) p(s) .
\end{aligned}
$$

Integrating both sides of inequality (3.4) gives

$$
\begin{gathered}
\int_{a}^{b} \int_{c}^{d}[\phi(f(t), h(s))-\phi(g(t), k(s))] w(t) p(s) d t d s \\
\geq \int_{a}^{b} \int_{c}^{d} \psi_{s}^{1}(t)[f(t)-g(t)] w(t) p(s) d t d s \\
\quad+\int_{a}^{b} \int_{c}^{d} \psi_{s}^{2}(t)[h(s)-k(s)] w(t) p(s) d t d s .
\end{gathered}
$$

By Fubini's theorem we have

$$
\begin{aligned}
& \int_{a}^{b} \int_{c}^{d}[\phi(f(t), h(s))-\phi(g(t), k(s))] w(t) p(s) d t d s \\
& \quad \geq \int_{c}^{d} p(s)\left[\int_{a}^{b} \psi_{s}^{1}(t) d F(t)\right] d s+\int_{a}^{b} w(t)\left[\int_{c}^{d} \psi_{s}^{2}(t) d G(s)\right] d t
\end{aligned}
$$


Using integration by parts, we obtain

$$
\begin{gathered}
\int_{a}^{b} \int_{c}^{d}[\phi(f(t), h(s))-\phi(g(t), k(s))] w(t) p(s) d t d s \\
\geq \int_{c}^{d} p(s)\left[\left.\psi_{s}^{1}(t) F(t)\right|_{a} ^{b}-\int_{a}^{b} \psi_{s}^{3}(t) g^{\prime}(t) F(t) d t\right] d s \\
\quad+\int_{a}^{b} w(t)\left[\left.\psi_{s}^{2}(t) G(s)\right|_{c} ^{d}-\int_{c}^{d} \psi_{s}^{4}(t) k^{\prime}(s) G(s) d s\right] d t
\end{gathered}
$$

which yields

$$
\begin{aligned}
& \int_{a}^{b} \int_{c}^{d}[\phi(f(t), h(s))-\phi(g(t), k(s))] w(t) p(s) d t d s \\
& \quad \geq-\int_{c}^{d} \int_{a}^{b} \psi_{s}^{3}(t) g^{\prime}(t) F(t) p(s) d t d s-\int_{a}^{b} \int_{c}^{d} \psi_{s}^{4}(t) k^{\prime}(s) G(s) w(t) d s d t
\end{aligned}
$$

Since $\phi$ is convex on $[0, \infty) \times[0, \infty)$, by Lemma 2.3 we conclude that $\phi$ is coordinate convex on $[0, \infty) \times[0, \infty)$, and thus $\psi_{s}^{3}(t) \geq 0, \psi_{s}^{4}(t) \geq 0$. Also, $k$ and $g$ are decreasing, so that $k^{\prime}(s) \leq 0$ and $g^{\prime}(t) \leq 0$. Thus it follows that

$$
-\int_{c}^{d} \int_{a}^{b} \psi_{s}^{3}(t) g^{\prime}(t) F(t) p(s) d t d s \geq 0
$$

and

$$
-\int_{a}^{b} \int_{c}^{d} \psi_{s}^{4}(t) k^{\prime}(s) G(s) w(t) d s d t \geq 0
$$

Combining (3.5), (3.6), and (3.7) yields

$$
\int_{a}^{b} \int_{c}^{d}[\phi(f(t), h(s))-\phi(g(t), k(s))] w(t) p(s) d t d s \geq 0,
$$

which implies the desired inequality (3.1).

(ii) Inequality (3.2) can be proved in the same way as inequality (3.1). Theorem 3.1 is proved.

Theorem 3.2 Let $w$ and $u$ be positive continuous functions on $[a, b]$ and $[c, d]$, respectively, and let $f, g$ and $k, l$ be positive differentiable functions on $[a, b]$ and $[c, d]$, respectively. Suppose that $\psi:[0, \infty) \times[0, \infty) \rightarrow \mathbb{R}$ is a convex function.

(i) Let $f / g$ and $l / k$ be decreasing functions on $[a, b]$ and $[c, d]$, respectively. If $f$ and $l$ are increasing functions on $[a, b]$ and $[c, d]$, respectively, then

$$
\begin{aligned}
& \int_{a}^{b} \int_{c}^{d} \psi\left(\frac{f(t)}{\int_{a}^{b} f(t) w(t) d t}, \frac{l(s)}{\int_{c}^{d} l(s) u(s) d s}\right) w(t) u(s) d t d s \\
& \quad \leq \int_{a}^{b} \int_{c}^{d} \psi\left(\frac{g(t)}{\int_{a}^{b} g(t) w(t) d t}, \frac{k(s)}{\int_{c}^{d} k(s) u(s) d s}\right) w(t) u(s) d t d s .
\end{aligned}
$$


If $g$ and $k$ are decreasing functions on $[a, b]$ and $[c, d]$, respectively, then the reverse inequality of (3.8) holds.

(ii) Let $f / g$ and $l / k$ be increasing functions on $[a, b]$ and $[c, d]$, respectively. If $g$ and $k$ are increasing functions on $[a, b]$ and $[c, d]$, respectively, then

$$
\begin{aligned}
& \int_{a}^{b} \int_{c}^{d} \psi\left(\frac{g(t)}{\int_{a}^{b} g(t) w(t) d t}, \frac{k(s)}{\int_{c}^{d} k(s) u(s) d s}\right) w(t) u(s) d t d s \\
& \quad \leq \int_{a}^{b} \int_{c}^{d} \psi\left(\frac{f(t)}{\int_{a}^{b} f(t) w(t) d t}, \frac{l(s)}{\int_{c}^{d} l(s) u(s) d s}\right) w(t) u(s) d t d s .
\end{aligned}
$$

Iff and $l$ are decreasing functions on $[a, b]$ and $[c, d]$, respectively, then the reverse inequality of (3.9) holds.

\section{Proof}

(i) Using Lemma 2.4 with substitution $v(t)=g(t) w(t)$ and $h(t)=f(t) / g(t)$ in (2.3), we obtain

$$
\begin{aligned}
& \int_{a}^{b} f(t) w(t) d t \int_{a}^{x} g(t) w(t) d t \\
& \quad \leq \int_{a}^{x} f(t) w(t) d t \int_{a}^{b} g(t) w(t) d t, \quad x \in[a, b] .
\end{aligned}
$$

Also, putting $v(s)=k(s) u(s)$ and $h(s)=l(s) / k(s)$ in (2.3) gives

$$
\int_{c}^{d} l(s) u(s) d s \int_{c}^{y} k(s) u(s) d s \leq \int_{c}^{y} l(s) u(s) d s \int_{c}^{d} k(s) u(s) d s, \quad y \in[c, d]
$$

From (3.10) and (3.11) we deduce that

$$
\begin{aligned}
& \int_{a}^{x}\left(\frac{g(t)}{\int_{a}^{b} g(t) w(t) d t}\right) w(t) d t \leq \int_{a}^{x}\left(\frac{f(t)}{\int_{a}^{b} f(t) w(t) d t}\right) w(t) d t, \quad x \in[a, b], \\
& \int_{c}^{y}\left(\frac{k(s)}{\int_{c}^{d} k(s) u(s) d s}\right) u(s) d s \leq \int_{c}^{x}\left(\frac{l(s)}{\int_{c}^{d} l(s) u(s) d s}\right) u(s) d s, \quad y \in[c, d] .
\end{aligned}
$$

Additionally, it is easy to observe that

$$
\begin{aligned}
& \int_{a}^{b}\left(\frac{g(t)}{\int_{a}^{b} g(t) w(t) d t}\right) w(t) d t=\int_{a}^{b}\left(\frac{f(t)}{\int_{a}^{b} f(t) w(t) d t}\right) w(t) d t, \\
& \int_{c}^{d}\left(\frac{k(s)}{\int_{c}^{d} k(s) u(s) d s}\right) u(s) d s=\int_{c}^{d}\left(\frac{l(s)}{\int_{c}^{d} l(s) u(s) d s}\right) u(s) d s .
\end{aligned}
$$

By relations (3.12), (3.13), (3.14), and (3.15), using inequality (3.2) given in Theorem 3.1, we obtain

$$
\int_{a}^{b} \int_{c}^{d} \psi\left(\frac{f(t)}{\int_{a}^{b} f(t) w(t) d t}, \frac{l(s)}{\int_{c}^{d} l(s) u(s) d s}\right) w(t) u(s) d t d s
$$




$$
\leq \int_{a}^{b} \int_{c}^{d} \psi\left(\frac{g(t)}{\int_{a}^{b} g(t) w(t) d t}, \frac{k(s)}{\int_{c}^{d} k(s) u(s) d s}\right) w(t) u(s) d t d s
$$

which is the desired inequality (3.8).

Using the majorization relations (3.12), (3.13), (3.14), and (3.15) and inequality (3.1), we get the reversed inequality of (3.8).

(ii) If we perform an interchange $f \longmapsto g(g \longmapsto f)$ and $k \longmapsto l(l \longmapsto k)$, then inequality (3.9) follows immediately from (3.8). The reversed inequality of (3.9) can be deduced from the reversed inequality of (3.8) by using the same interchange. This completes the proof of Theorem 3.2.

\section{Applications to the generalization of Favard's inequality}

As an application of Theorem 3.2, we establish some Favard-type inequalities for functions defined on rectangles, which generalize the results of Theorem 1.4 described in the Introduction.

Corollary 4.1 Let w and u be positive continuous functions on $[a, b]$ and $[c, d]$, respectively, and let $f$ and $l$ be positive differentiable functions on $[a, b]$ and $[c, d]$, respectively. Suppose that $\psi:[0, \infty) \times[0, \infty) \rightarrow \mathbb{R}$ is a convex function.

(i) Let $f(t) /(t-a)$ and $l(s) /(s-c)$ be decreasing functions on $(a, b]$ and $(c, d]$, respectively. If $f$ and $l$ are increasing functions on $[a, b]$ and $[c, d]$, respectively, then

$$
\begin{aligned}
\int_{a}^{b} & \int_{c}^{d} \psi(f(t), l(s)) w(t) u(s) d t d s \\
\leq & \int_{a}^{b} \int_{c}^{d} \psi\left(\frac{\int_{a}^{b} f(t) w(t) d t}{\int_{a}^{b}(t-a) w(t) d t}(t-a),\right. \\
& \left.\frac{\int_{c}^{d} l(s) u(s) d s}{\int_{c}^{d}(s-c) u(s) d s}(s-c)\right) w(t) u(s) d t d s .
\end{aligned}
$$

(ii) Let $f(t) /(b-t)$ and $l(s) /(d-s)$ be increasing function on $[a, b)$ and $[c, d)$, respectively. Iff and $l$ are decreasing functions on $[a, b]$ and $[c, d]$, respectively, then

$$
\begin{aligned}
\int_{a}^{b} & \int_{c}^{d} \psi(f(t), l(s)) w(t) u(s) d t d s \\
\leq & \int_{a}^{b} \int_{c}^{d} \psi\left(\frac{\int_{a}^{b} f(t) w(t) d t}{\int_{a}^{b}(b-t) w(t) d t}(b-t),\right. \\
& \left.\frac{\int_{c}^{d} l(s) u(s) d s}{\int_{c}^{d}(d-s) u(s) d s}(d-s)\right) w(t) u(s) d t d s .
\end{aligned}
$$

Proof Note the simple fact that if $\psi(x, y)(\psi:[0, \infty) \times[0, \infty) \rightarrow \mathbb{R})$ is a convex function, then $\psi\left(\theta_{1} x, \theta_{2} y\right)\left(\theta_{1}, \theta_{2}>0\right)$ is also a convex function. Using Theorem 3.2 with substitution

$$
\psi(x, y) \longmapsto \psi\left(\left(\int_{a}^{b} f(t) w(t) d t\right) x,\left(\int_{c}^{d} l(s) u(s) d s\right) y\right)
$$


in inequality (3.8) and choosing $g(t)=t-a$ and $k(s)=s-c$, we get the required inequality (4.1). Applying the above substitution to the reverse inequality of (3.9) and choosing $g(t)=$ $b-t$ and $k(s)=d-s$, we derive inequality (4.2).

Corollary 4.2 Let w and u be positive continuous functions on $[a, b]$ and $[c, d]$, respectively, and let $f$ and $l$ be positive differentiable functions on $[a, b]$ and $[c, d]$, respectively. Suppose that $\psi:[0, \infty) \times[0, \infty) \rightarrow \mathbb{R}$ is a convex function.

(i) Let $f(t) /(t-a)$ and $l(s) /(s-c)$ be decreasing functions on $(a, b]$ and $(c, d]$, respectively. If $f$ and $l$ are increasing functions on $[a, b]$ and $[c, d]$, respectively, then

$$
\begin{aligned}
& \frac{1}{(b-a)(d-c)} \int_{a}^{b} \int_{c}^{d} \psi(f(t), l(s)) w(t) u(s) d t d s \\
& \leq \int_{0}^{1} \int_{0}^{1} \psi(\xi \tilde{f}, \tilde{\eta}) w(a(1-\xi)+b \xi) u(c(1-\eta)+d \eta) d \xi d \eta,
\end{aligned}
$$

where $\widetilde{f}=(b-a) \int_{a}^{b} f(t) w(t) d t / \int_{a}^{b}(t-a) w(t) d t$ and $\widetilde{l}=(d-c) \int_{c}^{d} l(s) u(s) d s / \int_{c}^{d}(s-c) u(s) d s$.

(ii) Let $f(t) /(b-t)$ and $l(s) /(d-s)$ be increasing functions on $[a, b)$ and $[c, d)$, respectively. If $f$ and $l$ are decreasing functions on $[a, b]$ and $[c, d]$, respectively, then

$$
\begin{aligned}
& \frac{1}{(b-a)(d-c)} \int_{a}^{b} \int_{c}^{d} \psi(f(t), l(s)) w(t) u(s) d t d s \\
& \leq \int_{0}^{1} \int_{0}^{1} \psi(\xi \widetilde{f}, \eta \widetilde{l}) w(a \xi+b(1-\xi)) u(c \eta+d(1-\eta)) d \xi d \eta,
\end{aligned}
$$

where $\widetilde{f}=(b-a) \int_{a}^{b} f(t) w(t) d t / \int_{a}^{b}(b-t) w(t) d t$ and $\widetilde{l}=(d-c) \int_{c}^{d} l(s) u(s) d s / \int_{c}^{d}(d-s) u(s) d s$.

Proof Substituting $\xi=\frac{t-a}{b-a}$ and $\eta=\frac{s-c}{d-c}$ into the right-hand sides of (4.1), we get

$$
\begin{gathered}
\int_{a}^{b} \int_{c}^{d} \psi\left(\frac{\int_{a}^{b} f(t) w(t) d t}{\int_{a}^{b}(t-a) w(t) d t}(t-a), \frac{\int_{c}^{d} l(s) u(s) d s}{\int_{c}^{d}(s-c) u(s) d s}(s-c)\right) w(t) l(s) d t d s \\
=\int_{0}^{1} \int_{0}^{1} \psi\left(\xi \frac{(b-a) \int_{a}^{b} f(t) w(t) d t}{\int_{a}^{b}(t-a) w(t) d t}\right. \\
\left.\eta \frac{(d-c) \int_{c}^{d} l(s) u(s) d s}{\int_{c}^{d}(s-c) u(s) d s}\right) w(a(1-\xi)+b \xi) l(c(1-\eta)+d \eta) d \xi d \eta
\end{gathered}
$$

which, together with inequality (4.1), leads to the desired inequality (4.3).

Similarly, we can deduce inequality (4.4) by substituting $\xi=\frac{b-t}{b-a}$ and $\eta=\frac{d-s}{d-c}$ into the right-hand sides of (4.2).

Corollary 4.3 Let wand $u$ be positive continuous functions on $[a, b]$ and $[c, d]$, respectively. Suppose that $\psi:[0, \infty) \times[0, \infty) \rightarrow \mathbb{R}$ is a convex function. 
(i) Iff and l are positive increasing concave functions on $[a, b]$ and $[c, d]$, respectively, then

$$
\begin{aligned}
& \frac{1}{(b-a)(d-c)} \int_{a}^{b} \int_{c}^{d} \psi(f(t), l(s)) w(t) u(s) d t d s \\
& \leq \int_{0}^{1} \int_{0}^{1} \psi(\xi \tilde{f}, \eta \tilde{l}) w(a(1-\xi)+b \xi) u(c(1-\eta)+d \eta) d \xi d \eta
\end{aligned}
$$

where $\tilde{f}=(b-a) \int_{a}^{b} f(t) w(t) d t / \int_{a}^{b}(t-a) w(t) d t$ and $\tilde{l}=(d-c) \int_{c}^{d} l(s) u(s) d s / \int_{c}^{d}(s-c) u(s) d s$.

(ii) Iff and $l$ are positive decreasing concave functions on $[a, b]$ and $[c, d]$, respectively, then

$$
\begin{aligned}
& \frac{1}{(b-a)(d-c)} \int_{a}^{b} \int_{c}^{d} \psi(f(t), l(s)) w(t) u(s) d t d s \\
& \leq \int_{0}^{1} \int_{0}^{1} \psi(\xi \tilde{f}, \eta \tilde{l}) w(a \xi+b(1-\xi)) u(c \eta+d(1-\eta)) d \xi d \eta,
\end{aligned}
$$

where $\widetilde{f}=(b-a) \int_{a}^{b} f(t) w(t) d t / \int_{a}^{b}(b-t) w(t) d t$ and $\tilde{l}=(d-c) \int_{c}^{d} l(s) u(s) d s / \int_{c}^{d}(d-s) u(s) d s$.

Proof

(i) By the first part of Corollary 4.2, to prove inequality (4.5), it suffices to prove that $f(t) /(t-a)$ and $l(s) /(s-c)$ are decreasing functions on $(a, b]$ and $(c, d]$, respectively.

Consider the function

$$
P(t)=\frac{f(t)}{t-a}, \quad t \in(a, b] .
$$

Differentiation of $P(t)$ with respect to $t$ gives

$$
\begin{aligned}
P^{\prime}(t) & =\frac{f^{\prime}(t)(t-a)-f(t)}{(t-a)^{2}} \\
& =\frac{f^{\prime}(t)(t-a)-f(t)+f(a)}{(t-a)^{2}}-\frac{f(a)}{(t-a)^{2}} \\
& =\frac{f^{\prime}(t)-\frac{f(t)-f(a)}{t-a}}{t-a}-\frac{f(a)}{(t-a)^{2}} \\
& =\frac{f^{\prime}(t)-f^{\prime}(\theta)}{t-a}-\frac{f(a)}{(t-a)^{2}} \quad(a<\theta<t) .
\end{aligned}
$$

It follows that $P^{\prime}(t)<0$ for $t \in(a, b]$ since $f$ is a positive concave function on $[a, b]$. Thus we conclude that $P(t)=f(t) /(t-a)$ is a decreasing function on $(a, b]$. In the same way, we can prove that $l(s) /(s-c)$ is a decreasing function on $(c, d]$. This proves inequality (4.5).

(ii) Similarly, we have

$$
Q^{\prime}(t)=\left(\frac{f(t)}{b-t}\right)^{\prime}=\frac{f^{\prime}(t)-f^{\prime}(\zeta)}{b-t}+\frac{f(a)}{(b-t)^{2}} \quad(t<\zeta<b)
$$


We deduce that $Q^{\prime}(t)>0$ for $t \in[a, b)$, since $f$ is positive concave function on $[a, b]$. Thus, $Q(t)=f(t) /(b-t)$ is an increasing function on $[a, b)$. In the same way, we can prove that $l(s) /(d-s)$ is an increasing function on $[c, d)$. Therefore inequality (4.6) follows from the second part of Corollary 4.2.

\section{Final remarks}

Obviously, the results of Corollary 4.3 are generalizations of those given in Theorem 1.6 relating to Favard's inequality. Indeed, if we put $w(t)=1, u(s)=1, f=\Phi$, and $\psi(x, y)=\Upsilon(x)$ in (4.5) and (4.6), respectively, then we obtain the Favard inequality (1.6). Further, we can deduce the Favard inequality (1.7) by taking $\Upsilon(x)=x^{q}(q>1)$. It is worth noting that the majorization inequalities asserted in Theorem 3.1 play a key role in proving Theorem 3.2. Further, with the help of Theorem 3.2, we obtain some significant results in Corollaries $4.1,4.2$, and 4.3 .

\section{Funding}

This research was supported by funds from the International Scientific Research Publications (ISRP) under Grant No. 20180101R and the Natural Science Foundation of Fujian province of China under Grant No. 2016J01023.

\section{Competing interests}

The authors declare that they have no competing interests.

\section{Authors' contributions}

SW and MAK finished the proofs of the main results and the writing work. AB and RS gave lots of advice on the proofs of the main results and the writing work. All authors read and approved the final manuscript.

\section{Author details}

'Department of Mathematics, Longyan University, Longyan, China. ²Department of Mathematics, University of Peshawar, Peshawar, Pakistan. ${ }^{3}$ Department of Mathematics, Iran University of Science and Technology, Tehran, Iran.

\section{Publisher's Note}

Springer Nature remains neutral with regard to jurisdictional claims in published maps and institutional affiliations.

Received: 20 March 2018 Accepted: 19 June 2018 Published online: 27 June 2018

\section{References}

1. Adil Khan, M., Khalid, S., Pečarić, J.: Refinements of some majorization type inequalities. J. Math. Inequal. 7(1), 73-92 (2013)

2. Adil Khan, M., Latif, N., Pečarić, J: Generalization of majorization theorem. J. Math. Inequal. 9(3), 847-872 (2015)

3. Adil Khan, M., Latif, N., Pečarić, J.: Majorization type inequalities via Green function and Hermite polynomial. J. Indones. Math. Soc. 22(1), 1-25 (2016)

4. Adil Khan, M., Latif, N., Perić, I., Pečarić, J.: On Sapogov's extension of Čebyšev's inequality and related results. Thai J. Math. 10(3), 617-633 (2012)

5. Adil Khan, M., Latif, N., Perić, l., Pečarić, J.: On majorization for matrices. Math. Balk. 27(1-2), 3-19 (2013)

6. Adil Khan, M., Niezgoda, M., Pečarić, J.: On a refinement of the majorization type inequality. Demonstr. Math. 44(1), 49-57 (2011)

7. Ando, T:: Majorization, doubly stochastic matrices, and comparison of eigenvalues. Linear Algebra Appl. 118, 163-248 (1989)

8. Barnett, N.S., Cerone, P., Dragomir, S.S.: Majorization, doubly stochastic matrices, and comparison of eigenvalues. Appl. Math. Lett. 22, 416-421 (2009)

9. Bhatia, R.: Matrix Analysis. Springer, New York (1997)

10. Chu, Y.M., Wang, G.D., Zhang, X.H.: The Schur multiplicative and harmonic convexities of the complete symmetric function. Math. Nachr. 284(5-6), 653-663 (2011)

11. Chu, Y.M., Wang, M.K.: Optimal Lehmer mean bounds for the Toader mean. Results Math. 61(3-4), 223-229 (2012)

12. Chu, Y.M., Wang, M.K., Qiu, S.L.: Optimal combinations bounds of root-square and arithmetic means for Toader mean. Proc. Indian Acad. Sci. Math. Sci. 122(1), 41-51 (2012)

13. Chu, Y.M., Xia, W.F., Zhang, X.H.: The Schur concavity, Schur multiplicative and harmonic convexities of the second dual form of the Hamy symmetric function with applications. J. Multivar. Anal. 105, 412-421 (2012)

14. Chu, Y.M., Zhang, X.M.: Necessary and sufficient conditions such that the extended mean values are Schur-convex or Schur-concave. J. Math. Kyoto Univ. 48(1), 229-238 (2008)

15. Chu, Y.M., Zhang, X.M., Wang, G.D.: The Schur geometric convexity of the extended mean values. J. Convex Anal. 15(4), 707-718 (2008)

16. Dragomir, S.S.: On the Hadamard's inequality for convex functions on the co-ordinates in a rectangle from the plane. Taiwan. J. Math. 5(4), 775-778 (2001) 
17. Dragomir, S.S.: Some majorization type discrete inequalities for convex functions. Math. Inequal. Appl. 7(2), 207-216 (2004)

18. Favard, J.: Sur les valeurs moyennes. Bull. Sci. Math. 57(2), 54-64 (1933)

19. Fuchs, L.: A new proof of an inequality of Hardy-Littlewood-Pólya. Mat. Tidssker 13, 53-54 (1947)

20. Hardy, G.H., Littlewood, J.E., Pólya, G.: Inequalities, 2nd edn. Cambridge Univ. Press, Cambridge (1952)

21. Inoan, D., Rasa, I.: A majorization inequality for Wright-convex functions revisited. Aequ. Math. 83(3), 209-214 (2012)

22. Kadelburg, Z., Dukić, D., Lukić, M., Matić, I.: Inequalities of Karamata, Schur and Muirhead, and some applications. Teach. Math. VIII(1), 31-45 (2005)

23. Karamata, J.: Sur une inégalité relative aux fonctions convexes. Publ. Math. Univ. Belgrade 1, 145-148 (1932)

24. Karlin, S., Studden, W.J.: Tchebycheff Systems: With Applications in Analysis and Statistics. Interscience, New York (1966)

25. Kemperman, J.H.B.: Review: Albert W. Marshall and Ingram Olkin, Inequalities: Theory of majorization and its applications, and Y. L. Tong, Probability inequalities in multivariate distributions. Bull. Am. Math. Soc. 5(3), 319-324 (1981)

26. Latif, N., Pečarić, J., Perić, l.: On majorization, Favard's and Berwald's inequalities. Ann. Funct. Anal. 2(1), 31-50 (2011)

27. Maligranda, L., Pečarić, J., Persson, L.E.: Weighted Favard's and Berwald's inequalities. J. Math. Anal. Appl. 190, 248-262 (1995)

28. Marshall, A.W., Olkin, I.: Inequalities: The Theory of Majorization and Its Applications. Academic Press, New York (1979)

29. Marshall, A.W., Olkin, I.: Inequalities for the trace function. Aequ. Math. 29(1), 36-39 (1985)

30. Niezgoda, M.: Remarks on convex functions and separable sequences. Discrete Math. 308(10), 1765-1773 (2008)

31. Niezgoda, M.: Inequalities for convex sequences and nondecreasing convex functions. Aequ. Math. 91(1), 1-20 (2017)

32. Pečarić, J.: On some inequalities for functions with nondecreasing increments. J. Math. Anal. Appl. 98(1), 188-197 (1984)

33. Pečarić, J., Proschan, F., Tong, Y.L.: Convex Functions, Partial Orderings and Statistical Applications. Academic Press, New York (1992)

34. Qian, W.M., Chu, Y.M.: Sharp bounds for a special quasi-arithmetic mean in terms of arithmetic and geometric means with two parameters. J. Inequal. Appl. 2017, Article ID 274 (2017)

35. Schur, l.: Über eine Klasse von Mittelbildungen mit Anwendungen die Determinanten-Theorie Sitzungsber. Berlin Math. Gesellschaft 22, 9-20 (1923)

36. Shi, H.-N., Bencze, M., Wu, S.-H., Li, D.-M.: Schur convexity of generalized Heronian means involving two parameters. J. Inequal. Appl. 2008, Article ID 879273 (2008)

37. Shi, H.N., Wu, S.H.: Majorized proof and refinement of the discrete Steffensen's inequality. Taiwan. J. Math. 11(4), 1203-1208 (2007)

38. Wang, M.K., Chu, Y.M.: Landen inequalities for a class of hypergeometric functions with applications. Math. Inequal. Appl. 21(2), 521-537 (2018)

39. Wang, M.K., Li, Y.M., Chu, Y.M.: Inequalities and infinite product formula for Ramanujan generalized modular equation function. Ramanujan J. 46(1), 189-200 (2018)

40. Wang, M.K., Qiu, S.L., Chu, Y.M.: Infinite series formula for Hübner upper bound function with applications to Hersch-Pfluger distortion function. Math. Inequal. Appl. 21(3), 629-648 (2018)

41. Wu, S.: Generalization and sharpness of power means inequality and their applications. J. Math. Anal. Appl. 312(2) 637-652 (2005)

42. $\mathrm{Wu}, \mathrm{S}$.: Some results on extending and sharpening the Weierstrass product inequalities. J. Math. Anal. Appl. 308(2), 689-702 (2005)

43. Wu, S., Debnath, L.: Inequalities for convex sequences and their applications. Comput. Math. Appl. 54(4), 525-534 (2007)

44. Wu, S.H., Shi, H.N.: A relation of weak majorization and its applications to certain inequalities for means. Math. Slovaca 61(4), 561-570 (2011)

45. Wu, S.H., Shi, H.N.: Schur convexity of the generalized geometric Bonferroni mean and the relevant inequalities J. Inequal. Appl. 2018, Article ID 8 (2018)

46. Yang, Z.H., Chu, Y.M.: A monotonicity property involving the generalized elliptic integral of the first kind. Math Inequal. Appl. 20(3), 729-735 (2017)

47. Yang, Z.H., Qian, W.M., Chu, Y.M., Zhang, W.: On approximating the error function. Math. Inequal. Appl. 21(2), 469-479 (2018)

48. Yang, Z.H., Qian, W.M., Chu, Y.M., Zhang, W.: On approximating the arithmetic-geometric mean and complete elliptic integral of the first kind. J. Math. Anal. Appl. 462(2), 1714-1726 (2018) 